\title{
Development of a neutron detector for A1@MAMI
}

\author{
Michaela Thiel* \\ Institut für Kernphysik, Johannes Gutenberg-Universität Mainz, \\ Johann-Joachim-Becher-Weg 45, 55128 Mainz, Germany \\ E-mail: thiel@kph.uni-mainz.de
}

\section{Matthias Schoth}

Institut für Kernphysik, Johannes Gutenberg-Universität Mainz, Johann-Joachim-Becher-Weg 45, 55128 Mainz, Germany

E-mail: schoth@kph.uni-mainz.de

\section{on behalf of the A1 collaboration}

With the A1/KAOS spectrometers at the Institut für Kernphysik in Mainz high precision experiments are performed to investigate the structure of the nucleus and its constituents. So far the knowledge of the neutron form factor (FF) is limited because of poor detection efficiencies. The novel neutron detector aims for a neutron detection efficiency better than $80 \%$. Leading to smaller systematic uncertainties, it should be possible to improve measurements of the neutron electric $\mathrm{FF}$, especially in the low momentum tranfer range $\mathrm{Q}^{2}=0.2-1.5 \mathrm{GeV}^{2}$. In addition the new detector opens the possibility to study non-mesonic two-body weak decays. The concept of the detector foresees a modular concept with an active detector volume of about one cubic meter. To allow for high beam currents and the resulting high rates, the detector will be highly segmented using 48 crossed layers consisting of 48 bars with the dimension $\left(20\right.$ x 20 x 960) $\mathrm{mm}^{3}$. In total 4608 channels have to be read out via WLS fibers using silicon multipixel photon counters (MPPC). Performing a GEANT simulation, where the crossed layer geometry of the detector is taken into account, a spatial resolution of less than $1.5 \mathrm{~cm}$ (FWHM) and timing resolution of less than $0.5 \mathrm{~ns}$ is achievable. Several scintillator materials like liquids, solids or extrusions together with different WLS fibers have been tested and compared. The results from first prototype tests are presented.

50th International Winter Meeting on Nuclear Physics

23-27 January 2012

Bormio, Italy

\footnotetext{
*Speaker.
} 


\section{Introduction}

To investigate the structure of the nucleus and its constituents high precision experiments are needed. The aim is to learn more about the spatial charge- and current distribution inside the nucleon which are directly connected to the electromagnetic form factors. While for the proton the electric and magnetic form factor are well known (see i.e. [1],[2]), the determination of the neutron form factors is more demanding. First of all there are no free neutron targets available and secondly, the neutron has no net electric charge which makes the detection and identification in a detector system challenging. For an observation of the neutron it has to interact with the detector material it passes through. Afterwards, the charged particles which have been produced in those nuclear reaction can be detected. Unfortunately, the probability for such a conversion is only in the order of $1 \% / \mathrm{cm}$ and therefore limiting the statistics of the present data. Hence, the purpose is to build a neutron detector with a detection efficiency close to $100 \%$.

The aim is the adoption of the novel neutron detector together with the three high resolution spectrometers of the A1 collaboration at the Institut für Kernphysik, Mainz. Performing electron scattering experiments, precise data on the electric and magnetic form factor of the neutron should be provided.

\section{The neutron detector design}

As already mentioned in the last section, the detection of neutrons is challenging. Therefore the design of the detector has to fulfill certain requirements. The most important needs for the detector system can be summarized as follows:

1. large active detector volume

2. high segmentation

3. versatility

4. detection efficiency $>80 \%$

5. time resolution $<0.5 \mathrm{~ns}$

6. low costs

The first three points mainly constrain the geometry of the detector. A large active volume is needed to get a high detection efficiency, which should be at least $80 \%$. The aim is to have an active volume in the order of $1 \mathrm{~m}^{3}$. However, in a large detector volume the background contribution will rise, especially at higher beam currents $(\mathrm{I}>20 \mu \mathrm{A})$ which are needed in order to get sufficient statistics. This can be prevented by using a highly segmented system. Furthermore, the segmentation of the detector apparatus allows the usage in various experiments with different spatial arrangements. Thus, a modular system have been chosen, where the scintillator material is segmented into bars with the dimensions $(20 \times 20 \times 960) \mathrm{mm}^{3}$. These bars will be organized in layers, each containing 48 bars. Putting 48 layers one after another in a crossed geometry (see Figure 1) will cause a cubic detector volume in the end, comprising 2304 bars. Nevertheless, it will be possible to arrange the bars e.g. in a two fold geometry where a larger solid angle can be covered, but the depth of the detector volume is reduced.

To achieve a time resolution less than one nanosecond, a fast detector material is essential which can 


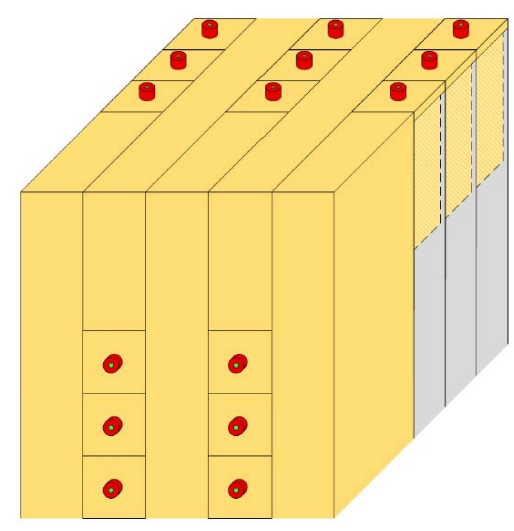

Figure 1: A section of the overall geometry of the neutron detector. In total, 48 layers - each consisting of 48 bars - are orientated perpendicular to each other and read out on both sides with a photosensor. In between the bars brass plates can be seen. (The figure is taken from [3].)

be realized by using plastic scintillators. Since polished plastic scintillator bars are very expensive, liquid scintillators were tested as an option to keep the costs as low as possible.

The scintillator bar should be read out via wavelength shifting fibers (WLS fibers) and multi pixel photon counters (MPPCs) at both sides. That implies a final read out of 4608 channels.

Taking all these constraints into account, different prototypes concerning detector material, fibers and photosensors have been studied. A description of the components and different setups tested will be given in the next sections.

\subsection{The scintillator material}

To find a good agreement between price and performance, four different scintillator materials have been studied. An overview of the tested materials is given in Table 1.

\begin{tabular}{|c|c|c|c|c|c|}
\hline scintillator & material & dimensions & em. peak & decay time & light output \\
\hline \hline BC-404 & plastic & $(20 \times 20 \times 960) \mathrm{mm}^{3}$ & $408 \mathrm{~nm}$ & $1.8 \mathrm{~ns}$ & $68 \%$ \\
\hline BC-490 & casted resign & $(20 \times 20 \times 410) \mathrm{mm}^{3}$ & $425 \mathrm{~nm}$ & $2.3 \mathrm{~ns}$ & $55 \%$ \\
\hline BC-519 & liquid oil & $(20 \times 20 \times 960) \mathrm{mm}^{3}$ & $425 \mathrm{~nm}$ & $4.0 \mathrm{~ns}$ & $60 \%$ \\
\hline EJ-309 & liquid & $(20 \times 20 \times 960) \mathrm{mm}^{3}$ & $424 \mathrm{~nm}$ & $3.5 \mathrm{~ns}$ & $75 \%$ \\
\hline
\end{tabular}

Table 1: The miscellaneous scintillators tested in various setups. The first three materials are from SaintGobain, the last one listed is from Eljen Technology. The given light output for all four materials is normalized to the light output of Anthracene.

To allow a placement of the WLS fiber for all kind of materials in the middle of the bar, the plastic scintillator has been delivered in two polished halves. So it was possible to cut a groove in one of the parts and afterwards glue the WLS fiber in the groove and both halves together with optical cement (EJ-500, Eljen Technology).

The casted resign was in a first step produced in house in one piece and in a second step cut into 
two halves with the groove in one of them. Then the fiber has been glued in and the both halves together in the same way it has been done for the plastic scintillator bars. Finally, the surfaces have been little polished. Unfortunately, due to the dimensions of the oven, where the casted resign had to heat up for two weeks, its length is $55 \mathrm{~cm}$ shorter compared to the other materials.

For the handling of the liquid scintillator aluminum housings with the same dimensions as the plastic scintillator bars have been built. To prevent a leakage of the material brass caps have been pressed at both ends into the aluminum housing.

\subsection{The wavelength shifting fiber}

A wavelength shifting (WLS) fiber is used to couple the active area of the photosensor $\left(1 \mathrm{~mm}^{2}\right)$ to the scintillator with a much larger surface of $(20 \times 20) \mathrm{mm}^{2}$. To fit the active area of the photosensor best, fibers with a quadratic cross-section surface have been chosen.

WLS fibers from two different vendors have been tested, whereas the main difference between both fibers is related to their cladding (see Table 2).

\begin{tabular}{|c|c|c|c|c|c|}
\hline WLS fiber & cladding & vendor & dimensions & absorp. peak & em. peak \\
\hline \hline BCf-92 & double & Saint-Gobain & $1 \mathrm{~mm}$ square & $410 \mathrm{~nm}$ & $492 \mathrm{~nm}$ \\
\hline Y-11 & single & Kuraray & $1 \mathrm{~mm}$ square & $430 \mathrm{~nm}$ & $476 \mathrm{~nm}$ \\
\hline
\end{tabular}

Table 2: The used wavelength shifting fibers tested in various setups.

With the double cladded fibers from Saint-Gobain it also has been studied whether the position of the fiber in the scintillator material plays a role for the amount of detected photons. Therefore different caps, shown in Figure 2 (left), have been produced. They are attached to the end faces of the solid scintillators (see Figure 2, middle) and should ensure the stabilization of the fiber as well as a good coupling to the photosensor.

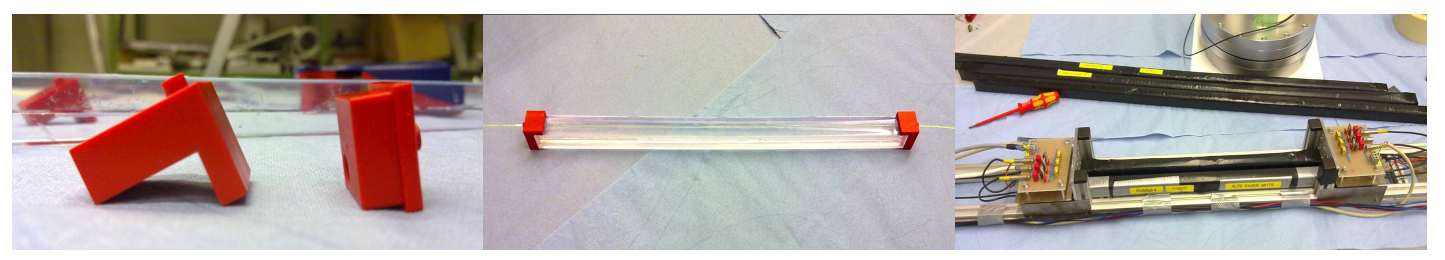

Figure 2: Left: For stabilization of the WLS fiber and their coupling to the photosensor two different caps have been constructed: one where the fiber is placed in the middle and one where the fiber is placed at the side of a bar. Middle: One plastic scintillator bar with the WLS fiber placed at the side and the corresponding caps attached to the end faces of the bar. Right: In the front the setup of two prototype bars composed of casted resign are shown. At both sides the readout electronics can be seen. The length can be compared to the bars shown in the back, which are made of plastic scintillator.

\subsection{The photosensor}

For the read out of the first prototype bars multi pixel photon counters (MPPCs) have been chosen based on the several advantages they have. The low bias voltage as well as the small housing (SMD package) allow an easy handling and guarantee the versatility of the detector system. 
Furthermore, one important aspect is the low prize per MPPC, because of the high quantity which is needed for 4608 read out channels. In addition, the photon amplification can be determined precisely from the dark count spectrum. With an automated online compensation of the bias voltage it is possible to stabilize the amplification very accurately.

One drawback of multi pixel photon counters is the risk of radiation damages during the experiment. In a separate setup this task has been studied during the first in-beam test.

\section{First test using the electron beam at the Mainz microtron MAMI}
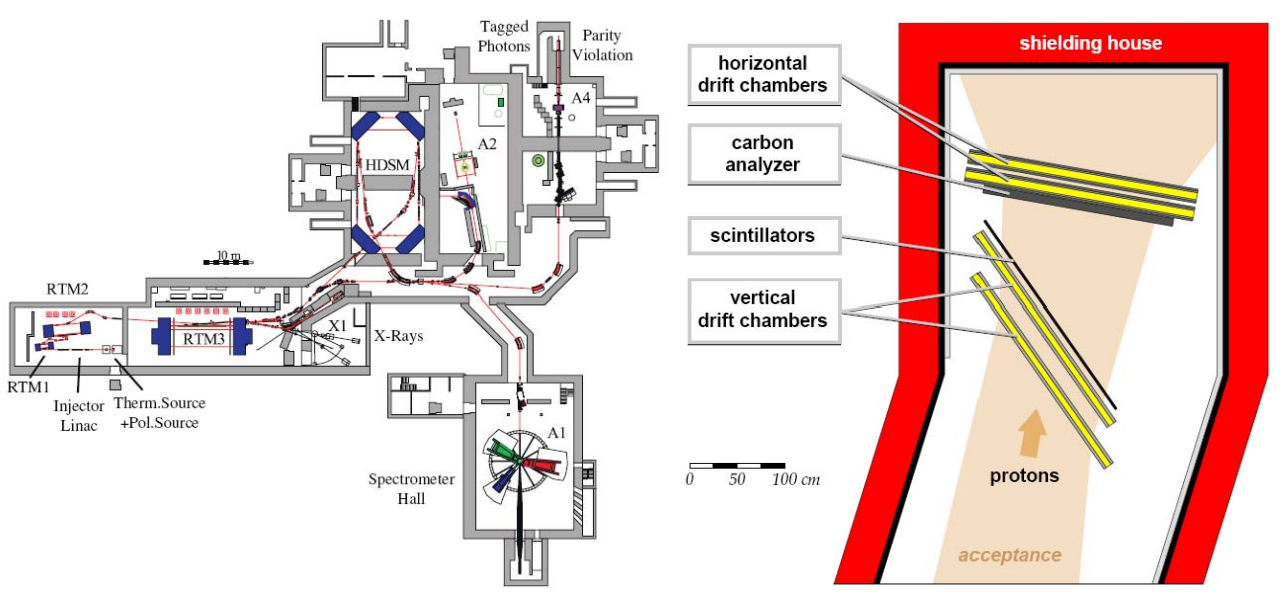

Figure 3: Left: Overview of the Mainz microtron MAMI with the three racetrack mictrotrons and the harmonic double sided microtron. In addition the experimental halls A1, A2, A4 and X1 are shown. (The figure is taken from [5].) Right: Schematic view of the high resolution spectrometer A. For the first beam test, the prototypes were placed between the scintillators and the upper vertical drift chamber. (The figure is taken from [6].)

The first in-beam tests have been performed at the electron accelerator facility MAMI, comprising of three racetrack microtrons (RTM) [4] and one harmonic double sided microtron (HDSM) [5]. The prototype test took place in the A1 spectrometer hall of the Institut für Kernphysik, JGU Mainz. An overview of the accelerator and the experimental halls is illustrated in Figure 3 left.

The different prototype bars (see Figure 2, right) were placed inside the high resolution spectrometer $A$ between the upper vertical drift chamber and the plastic scintillators of the spectrometer (see Figure 3, right). The existing detectors inside the spectrometer have been used to identify the particles impinging on the neutron detector, including time and spatial information.

One goal of the test experiment was to see the possible time resolution which can be achieved by detecting protons. Due to the chosen magnetic field of the spectrometer, the momentum of the proton has been restricted to $p_{p}=450 \mathrm{MeV} / \mathrm{c}$. The prototype tests were running with an electron beam energy of $E_{e-}=1508 \mathrm{MeV}$ and a beam current of $\mathrm{I}=10 \mu \mathrm{A}$.

The signals of the MPPCs have been amplified by a factor 10 with a low noise amplifier $(1.8 \mathrm{GHz}$ band width) which was particular designed for this purpose. Finally the signals have been read out with a Tektronix oscilloscope (TDS 3014B, $1.25 \mathrm{GS} / \mathrm{s}$ ), therefore only two bars could be read out 

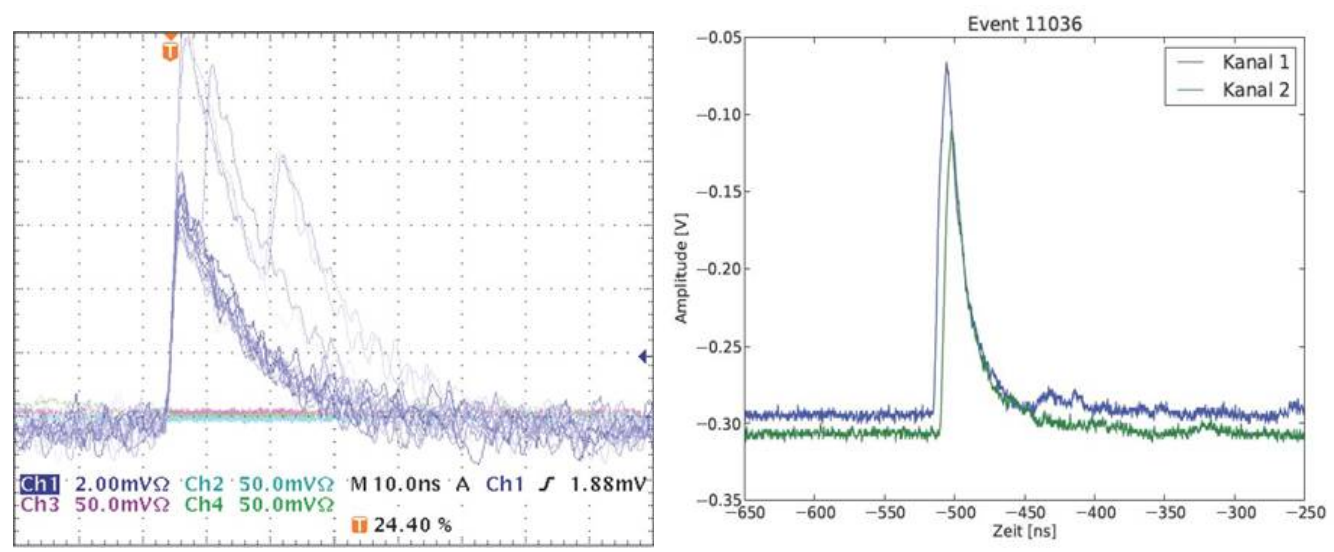

Figure 4: Left: The area of the recorded MPPC signal (after amplification) corresponding to a one-photon event. The larger signal belongs to a more-photon event. Right: Response of a scintillator bar: the signal in blue is read out at the left side, the green signal at the right side, both are in coincidence.

simultaneously. The data has been recorded with a rate of approximately $\mathrm{f}=0.5 \mathrm{~Hz}$ and more than 4000 events per prototype have been stored.

\section{Results}

Since the data has been recorded with an oscilloscope, the full lineshape information of the signals is available. Via the area of the signal the number of detected photons can be calculated. Using the known area for a one-photon signal $\left(\mathrm{A}_{p}=1.68 \mathrm{pC}\right)$ which is shown in Figure 4 left, the number of detected photons can be calculated:

$$
N_{p h}=\frac{\sqrt{A_{1} \cdot A_{2}}}{A_{p}}
$$

Here $A_{1}$ and $A_{2}$ correspond to the area of the signals read out at each side of a bar. The obtained results are summarized in the Tables 3,4 .

\begin{tabular}{|l||l|l|l|l|}
\hline \multicolumn{1}{|l||}{ Scintillator } & \multicolumn{2}{c|}{ BC-404 (plastic) } & \multicolumn{2}{c|}{ BC-490 (casting resign) } \\
\hline setup & BCF-92 (side) & BCF-92 (middle) & BCF-92 (side) & BCF-92 (middle) \\
\hline \hline$\Delta \mathbf{t}[\mathbf{n s}]$ & $0.89 \pm 0.05$ & $0.85 \pm 0.04$ & $0.90 \pm 0.04$ & $0.87 \pm 0.02$ \\
\hline $\mathbf{N}_{p h}$ & $32.78 \pm 0.30$ & $38.33 \pm 0.43$ & $21.65 \pm 0.36$ & $21.96 \pm 0.17$ \\
\hline
\end{tabular}

Table 3: The achieved time resolution and the corresponding number of detected photons for the various prototypes.

As the scintillator bars have been read out at both sides with an oscilloscope (Figure 4, right) the time difference and the impact position, which is determined with a spatial resolution of less than one millimeter using the vertical drift chamber in the spectrometer, can be used to reconstruct the impact time of the particle on the bar. Figure 5 shows the resulting distribution, corrected for the 


\begin{tabular}{|l||l|l|l|l|}
\hline \multicolumn{1}{|l||}{ Scintillator } & \multicolumn{2}{c|}{ BC-519 (liquid oil) } & \multicolumn{2}{c|}{ EJ-309 (liquid) } \\
\hline setup & new filling & old filling & BCF-92 (middle) & Y-11 (middle) \\
\hline \hline$\Delta \mathbf{t}[\mathbf{n s}]$ & $2.43 \pm 0.03$ & $2.81 \pm 0.03$ & $1.00 \pm 0.02$ & $1.46 \pm 0.02$ \\
\hline $\mathbf{N}_{p h}$ & $8.25 \pm 0.08$ & $5.88 \pm 0.05$ & $19.05 \pm 0.08$ & $18.45 \pm 0.09$ \\
\hline
\end{tabular}

Table 4: The achieved time resolution and the corresponding number of detected photons for the various prototypes.
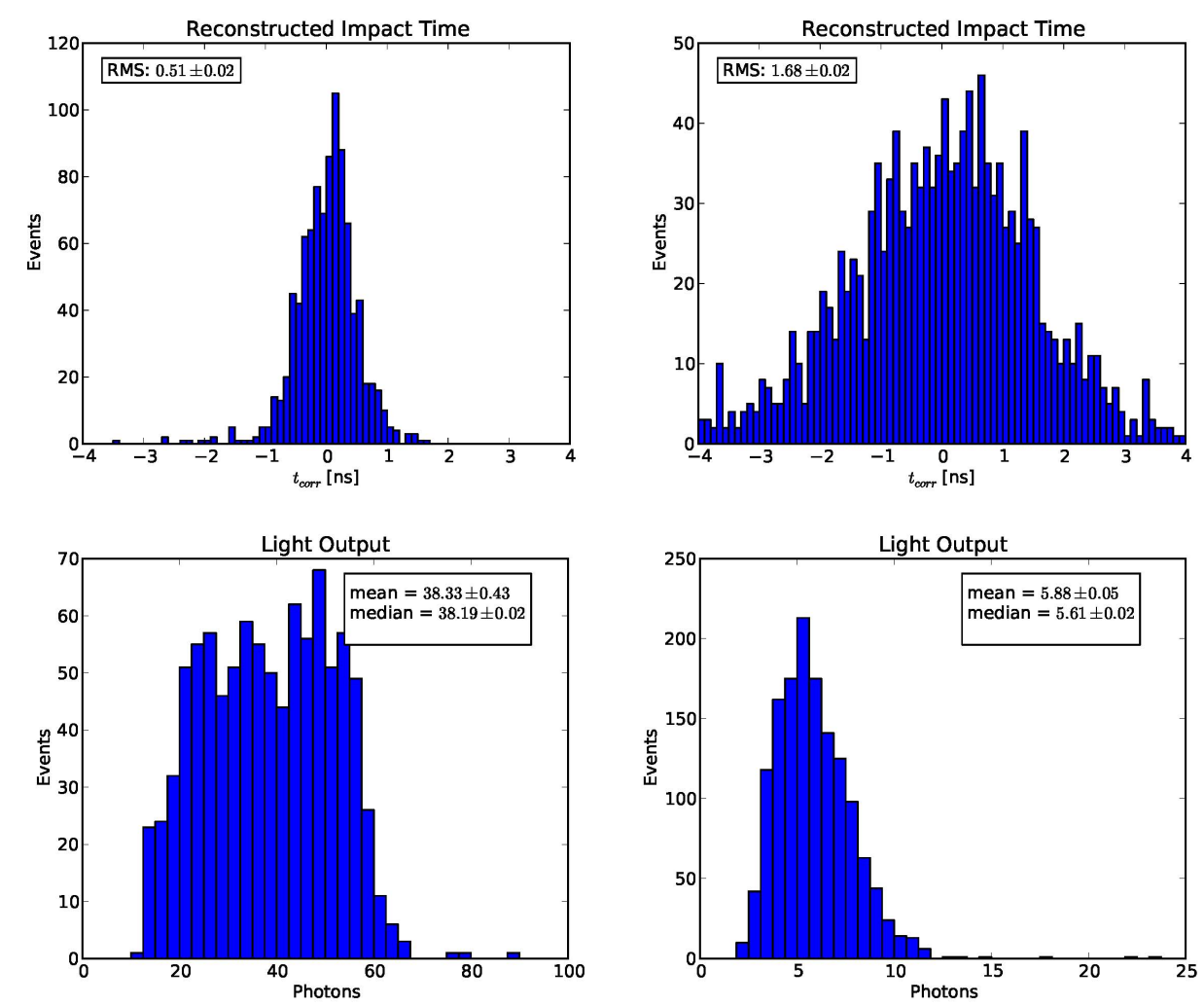

Figure 5: Upper row: Distribution of the reconstructed impact time for one bar; plastic scintillator with WLS fiber in the middle (left) and liquid scintillator with WLS fiber in the middle, filled 13 month ago (right). Lower row: Number of detected photons at one side of a bar; plastic scintillator with WLS fiber in the middle (left) and liquid scintillator with WLS fiber in the middle, filled 13 month ago (right).

position of impact and the effective speed of light along the detector. From this distribution the time resolution per channel (FWHM) can be calculated:

$$
\Delta t=R M S \cdot \frac{2 \sqrt{2 \ln (2)}}{\sqrt{2}},
$$

assuming the same resolution per channel for both sides. The results obtained for the different prototype setups are listed in the Tables 3, 4 .

The direct comparison of the different scintillator materials shows that the best time resolution can 
be obtained with plastic scintillators. A factor of three have been found between the best (plastic) and the worst (liquid oil, old) scintillator. Nevertheless it can be seen, that also the differences between various liquids are large. With the liquid scintillator from Eljen Technology the achieved time resolution is in the same order like the one for plastic scintillators.

Comparing the two positions where the fiber was located, it can be seen, that the combination with the fiber in the middle of the bar always gives a better time resolution compared to the ones obtained when the fiber is located at the side of the bar. The fiber with only single cladding is worse compared to the one with double cladding. The results for the time resolution and light output of the best and the worst combination are illustrated in Figure 5.

\section{Concluding remarks}

The first performance test for several prototype modules have been succesfully carried out at the Mainzer microtron MAMI. The combination of plastic scintillator with the WLS fiber placed in the middle of the bar delivers the best result. With a moderate number of detected photons, a time resolution of $\Delta \mathrm{t}=(0.89 \pm 0.05) \mathrm{ns}$ could be achieved. In addition, it has been observed that the time resolution is dependig on the number of detected photons.

In the next step a larger prototype will be build. Due to the experience made during the tests of the different configurations, it will contain plastic scintillators with double cladded WLS fibres. To further increase the light output on the active area of the photosensor, the number of fibers will be increased up to four per bar.

Since the additional test of the multi pixel photon counters shows severe damages of the MPPC due to radiation, the read out of the large prototype will consist of multi-anode PMTs (R7259K series from Hamamatsu Photonis, Japan). Due to the fact that these MaPMTs are already used in the fiber detector of the KAOS spectrometer at MAMI [7], one profits from existing resources and know-how.

\section{References}

[1] J.C. Bernauer et al., High-precision determination of the electric and magnetic form factors of the proton, Phys. Rev. Lett. 105 (2010) 242001

[2] X. Zhan et al., High Precision Measurement of the Proton Elastic Form Factor Ratio $\mu_{p} G_{E} / G_{M}$ at low $Q^{2}$, Phys. Lett. B 705 (2011) 59

[3] M. Rohrbeck, Ein neus Konzept für einen stark segmentierten Neutrondetektor, Masterarbeit, Johannes Gutenberg-Universität Mainz, Institut für Kernphysik, 2011

[4] H. Herminghaus et al., The design of a Cascaded $800 \mathrm{MeV}$ Normal Conducting CW Racetrack Microtron, Nucl. Instrum. Methods 138 (1976) 1

[5] K.-H. Kaiser et al., The $1.5 \mathrm{GeV}$ harmonic double-sided microtron at Mainz University, Nucl. Instr. Meth. A 593 (2008) 159

[6] Th. Pospischil et al., The focal plane proton-polarimeter for the 3-spectrometer setup at MAMI, Nucl. Instr. Meth. A 483 (2002) 713

[7] P. Achenbach et al., In-beam tests of scintillating fibre detectors at MAMI and at GSI, Nucl. Instr. Meth. A 593 (2008) 353 